\title{
The diagnostic value of the shoulder rotator cuff muscles' isometric force testing
}

\author{
Yaron Berkovitch", Maruan Haddad*, Yaniv Keren", Michael Soudry, Nahum Rosenberg ${ }^{\#}$ \\ Laboratory of Musculoskeletal Research, Rambam Health Care Campus, Haifa, Israel \\ Email: " nahumrosenberg@hotmail.com
}

Received 20 July 2013; revised 20 August 2013; accepted 28 August 2013

Copyright (C) 2013 Yaron Berkovitch et al. This is an open access article distributed under the Creative Commons Attribution License, which permits unrestricted use, distribution, and reproduction in any medium, provided the original work is properly cited.

\begin{abstract}
The most prevalent group of disorders of human shoulder is related to the muscles of the rotator cuff. In order to develop a mechanical method for rotator cuff muscles' evaluation, we hypothesized that measurement of the isometric force generated by the individual muscle of the rotator cuff might detect the variations, which are characteristic to the different disorders of rotator cuff muscles in adults. The isometric force of supraspinatus, infraspinatus and subscapularis muscles were measured in patients with rotator cuff tears, calcific tendinitis and subacromial impingement syndrome, 30 patients with each disorder, and compared to the normal values from our previous study. Torque of the force was calculated and normalized to lean body mass. The profiles of the mean torque-time curves of each group were compared statistically. We found the expected significantly lower profiles of the torque-time curves of all the tested rotator cuff muscles in comparison to the normal values. The best resolution between the curves of different study groups was found in the testing of the infraspinatus muscles. Therefore the previously unrecognized variations of rotator cuff muscles' isometric strength build up patterns in the common disorders involving the rotator cuff muscles were revealed. The presented data might be a basis for the future development of a simple mechanical diagnostic method for identification of the abnormal patterns of muscle isometric strength in patients with rotator cuff muscles' pathology.
\end{abstract}

Keywords: Shoulder; Muscle; Rotator Cuff; Isometric Force

\footnotetext{
*Equal contribution.

"Corresponding author.
}

\section{INTRODUCTION}

Disorders of shoulder which are related to the pathological processes in the muscles of rotator cuff are very common [1-4]. Pathology of these muscles is usually expressed by pain, weakness and restriction of shoulder movements. The diagnosis of rotator cuff muscles' pathology is usually based on an initial physical examination, which has a limited prediction values, and can be established with a high precision by much more sophisticated imaging modalities, like ultrasound and MRI scans. Ideally the ultimate cost effective diagnostic tool for this purpose should combine the simplicity and a low cost of the physical examination with the precision of the imaging tools. Since the mechanical force generated by these muscles is easily measurable, the pathology of these muscles might be identified by measurement of the building up of an isometric force by these muscles with comparison to the data in normal population. Till recently comprehensive data on the isometric force generation by the rotator cuff muscles in normal population, including variations according to gender, age and dominancy, were unavailable and the evaluation of rotator cuff pathology by measurement of the generated force was impossible because of the lack of reference for a comparison. Several previous attempts have been made to determine the normal values of the isometric force of the rotator cuff muscles [5-11], but these studies did not provide full data on a wide spectrum of ages in large study groups. In our recent initial study we developed and standardized a method for the evaluation of a buildup of an isometric force of individual rotator cuff muscles in normal adult population [12]. In that study, based on evaluation of shoulders of 400 normal volunteers, we recorded the variations of the profiles of torque-time curves of isometric force which are generated by supraspinatus (SS), infraspinatus (IS) and subscapularis (SSC) muscles according to the limb dominancy, age and gender. In the current study we used these data as a reference in order to evaluate patients with rotator cuff pathology by mechanical 
measurements.

In this project we hypothesize that by evaluating the isometric mechanical force generation by the shoulder rotator cuff muscle in patients with rotator cuff muscle disorders, a significant difference from the normal values, with specific pattern of difference for each type of pathology, will be revealed. Recognizing the existence of this difference will allow the future standardization of a simple clinical method for diagnosis of rotator cuff muscles' disorders using testing of isometric force generation.

In this project we investigated patients suffering from shoulder subacromial impingement, from shoulder calcific tendinitis and patients with grade 2 tears of SS.

Impingement syndrome is among the most common causes of shoulder pain in adults. The syndrome is caused by compression of the tendons of the rotator cuff muscles (most commonly the supraspinatus) between the coracoacromial arch (which includes the acromion, the coracoid process and the connecting coracoacromial ligament) and a humeral head [13]. Impingement causes microtrauma to the rotator cuff with resulting inflammation, pain and loss of function. Part of the reason for the development of the syndrome may be subacromial space narrowing which could be caused by osteophyte formation or by previous fractures. The source of this pathological process is extrinsic to the SS muscle and tendon.

Complete tears of SS and IS muscles are prevalent after age 55 - 60 years [14] and can be due to intrinsic muscle/tendon degeneration with or without contribution of traumatic event. The grade 2 complete tears are of intermediate extension, in diameter between $1.0-3.0 \mathrm{~cm}$ [15]. This type of tears is usually a source of disabling pain without compromising the ability of active shoulder movements [16].

Calcific tendonitis is of inflammatory origin and involves the rotator cuff muscles. Disabling shoulder pain is common due to this intrinsic inflammatory process, usually in SS and SSC tendons and muscles. This disorder becomes prevalent from the $3^{\text {rd }}$ decade of life.

The common clinical presentation of these three shoulder disorders, caused by the rotator cuff intrinsic or extrinsic pathology, is disabling pain in the shoulder area without compromise of the active shoulder movements. Currently the final diagnosis is based on imaging modalities. In the present study we will attempt to present a simple mechanical diagnostic method that might distinguish between these disorders, and potentially might be used as a simple tool for a diagnosis of rotator cuff muscles' pathology.

\section{METHODS}

\subsection{Study Group}

Three groups of patients were tested:

1) A group of 30 patients with known subacromial im- pingement syndrome, diagnosed clinically and supported by an ultrasound scan, without rotator cuff tears. The subjects were in the age between 30 - 65 years, 19 men and 11 women. In 19 of the patients the shoulder with impingement syndrome was affected in the dominant arm and in 11 patients the non-dominant arm was involved.

2) A group 30 patients with grade 2 complete tears in SS. The disorder was diagnosed clinically and supported by an ultrasound and/or MRI scans. The subjects were in the age between 50 - 75 years, 21 men and 9 women. In 25 of the patients the shoulder with impingement syndrome was in the dominant arm and in five in the nondominant arm.

3) A group 30 patients who suffer from shoulder pain due to calcific tendinitis in the rotator cuff muscles without rotator cuff tears. These patients were diagnosed clinically and supported by an ultrasound scan and radiographs. The subjects were in the age between $32-58$ years, 22 men and 8 women. In six of the patients the affected shoulder was in the dominant arm and 24 patients in the non-dominant arm.

Patients with neurological impairment of upper limbs, those with previous fractures of the shoulder and those who were unable to cooperate due to cognitive impairment were excluded from the study.

\subsection{Measurements}

Since most of the clinically important pathologic changes in rotator cuff muscles, e.g. tears, partial or full, degeneration, such in different impingement syndromes, calcific tendenitis, etc., occur in SS, IS and SSC muscles, these structures were evaluated in the present study.

Isometric force-time curves of the tested muscles in the affected shoulders in every person were measured at standardized positions. The dominancy of the tested limb was recorded. The measurements of the isometric force were performed by a specialized dynamometer (Atlantech@Nottingham Myometer, 1200 readings/sec, resolution $0.04 \mathrm{~N}$ ).

Overall rotator cuff muscles act synergistically, but there are several testing positions when one of these muscles generates the main force [17]. We used these optimal positions for the measurements:

- For the SS muscle testing the tested arm was held in $45 \mathrm{deg}$. of abduction and $30 \mathrm{deg}$. of forward flexion with the dynamometer cuff placed above elbow. The elbow was flexed to $90^{\circ}$. The lever arm of abduction force was measured between the center of the dynamometer cuff and the point $3 \mathrm{~cm}$ distal to the lateral edge of acromion that represents the center of rotation of the humeral head.

- The SSC and IS muscles were tested when the exam- 
ined individual is in a supine position with the tested extremity is $30^{\circ}$ tilted forward and fully adducted. This positioning is chosen in order to eliminate scapular movements and in order to evaluate the generated forces close to the scapular plane. The dynamometer cuff was placed on the forearm and the forces were produced by the internal and external rotation of the shoulder. The connection between the dynamometer cuff and the dynamometer was kept perpendicular to the forearm, in the opposite direction to the force vector and therefore the lever arm in this position is negligible.

Each strength measurement was performed for $5 \mathrm{sec}-$ onds time period. This period of the test was chosen empirically similarly to the widely accepted Constant's shoulder assessment scoring method [18]. Two measurements were performed for each muscle in five-minute intervals and the highest results were recorded. Characteristic forcetime curves were produced (Figure 1). For the further evaluation the values from the beginning of the generated force rise and in the duration of 4 seconds were used in order to eliminate the artifacts of the initiation and completion of measurements. Therefore the information was recorded as sequence of measurements lasting four seconds. Each value was multiplied by the lever arm and expressed as a moment of torque. The results were normalized to the lean body mass in order to eliminate the effect of the variations in the overall fitness conditions of the examined subjects. The lean body mass (LBM) was calculated according to the following [19]:

$$
\begin{aligned}
& \operatorname{LBM}(\text { men }) \\
& =(1.10 \times \text { Weight })-128 \times\left(\text { Weight }^{2} /\left(100 \times \text { Height }^{2}\right)\right) \\
& \text { LBM }(\text { women }) \\
& =(1.07 \times \text { Weight })-148 \times\left(\text { Weight }^{2} /\left(100 \times \text { Height }^{2}\right)\right)
\end{aligned}
$$

Average values for each group were recorded and standard deviations were calculated.

In order to verify that a maximal isometric strength was intentionally exerted, which is essential for the detection of force buildup impairment [20], surface EMG was recorded (Myotrace 400, Noraxon EMG \& Sensor Systems, Scottsdale, Arizona, USA) from the anterior deltoid and IS muscles as a crude presentation of the overall recruitment of the shoulder muscles for a maximal isometric effort (Figure 2).

\subsection{Statistical Analysis}

We studied the force-time curves from the three groups of patients described above and compared to the data of the matched, according to age, gender and dominancy, group of normal volunteers who were evaluated in our

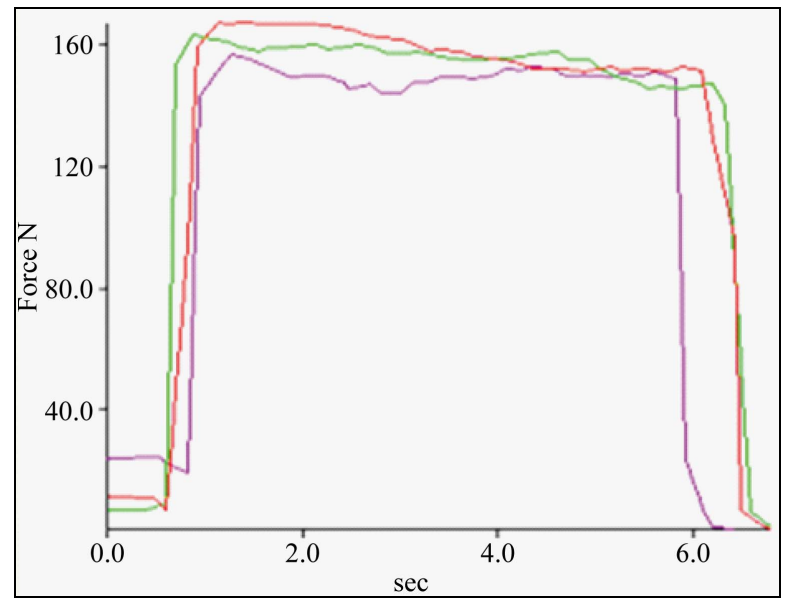

Figure 1. Examples of the profiles of the recorded by a dynamometer force-time curves.

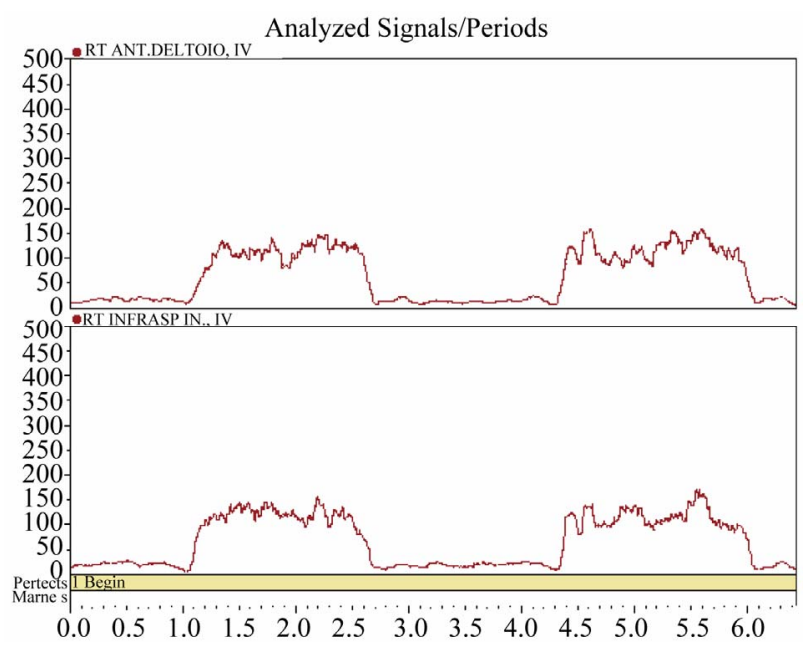

Figure 2. Examples of surface EMG recording from anterior deltoid and IS muscles showing an intentional recruitment of this muscles for a force generation.

previous study [12]. The corresponding values of force, according to the equal time intervals, were compared by One Way ANOVA test followed by posthoc Tukey test (for comparison of pairs of the tested groups) after a normal distribution of values was determined by the Kolmogorov-Smirnov test, otherwise One Way ANOVA on Ranks with Student Newman Keuls post-hoc test was used. $\mathrm{P}$ value of less than 0.05 was considered as statistically significant. The calculations of the statistical comparisons were performed by SigmaStat software (version 2, SPSS Inc., Chicago, IL, USA).

This project was approved by the Institutional Ethical Committee.

\section{RESULTS}

\subsection{Supraspinatus Muscle Testing}

In the testing of SS muscle we found a significantly lo- 
wer profiles of the torque time curves in all three pathological conditions in comparison to the normal values, i.e. average maximal torque in patients with subacromial impingement, calcific tendinitis and SS tears were 25.9 $+/-8.3,22.6+/-5.6$ and $22.9+/-5.5 \mathrm{~N}^{*} \mathrm{~m} / \mathrm{kg}$ respectively, vs. normal values of $61.8+/-18.3 \mathrm{~N} * \mathrm{~m} / \mathrm{kg}, \mathrm{p}<$ 0.001 , (Figure 3). The slope of force generation was similarly less steep in all these three conditions in comparison to the normal curve (Figure 3). The curves of patients with SS tears and calcifications were undistinguishable $(p>0.05)$ and significantly lower than the curve profile of patients with subacromial impingement syndrome $(\mathrm{p}<0.01)$.

\subsection{Infraspinatus Muscle Testing}

In the testing of IS muscle we found a significantly lower profiles of the torque, time curves in all three pathological conditions in comparison to the normal values, i.e. average maximal torque in patients with subacromial impingement, calcific tendinitis and SS tears were $20.3+/-$ $6.2,5.7+/-2.9$ and $26.3+/-6.5 \mathrm{~N}^{*} \mathrm{~m} / \mathrm{kg}$ respectively, vs. normal values of $40.4+/-10.4 \mathrm{~N}^{*} \mathrm{~m} / \mathrm{kg}, \mathrm{p}<0.001$, (Figure 4). The slopes of force generation were similarly less steep in all these three conditions in comparison to the normal curve (Figure 4). The curves and the slopes of force generation of patients with SS tears, calcific tendinitis (calc) and subacromial impingement (imp) were significantly distinguishable $(\mathrm{p}<0.01)$ in the following order: tear $>$ imp $>$ calc.

\subsection{Subscapularis Muscle Testing}

In the testing of SSC muscle we found a significantly lower profiles of the torque, time curves in patients with impingement syndrome and calcific tendinitis in compa- rison to normal values and curves of patients with SS tears, which were undistinguishable $(p>0.05)$, i.e. average maximal torque in patients with subacromial impingement and calcific tendinitis were $24.3+/-7.1$ and 6.4 $+/-1.9 \mathrm{~N}^{*} \mathrm{~m} / \mathrm{kg}$ respectively vs. normal values and curves of patients with SS tears, with $29.0+/-8.9$ and 30.4 $+/-7.0 \mathrm{~N}^{*} \mathrm{~m} / \mathrm{kg}$ respectively, $\mathrm{p}<0.001$ (Figure 5). The curves and the slopes of force generation of patients with SS tears, calcific tendinitis (calc) and subacromial impingement (imp) were significantly distinguishable $(\mathrm{p}<0.01)$ in the following order: tear $>$ imp $>$ calc.

\section{DISCUSSION}

The use of a portable dynamometer for measurement of isometric muscle force has been established previously [21]. In our previous study we utilized this method for determination of the normal values of isometric force generated by the rotator cuff muscles [12] and developed a database of normal values of isometric force generation by these muscles. Accordingly in the present study we were able to detect the differences of the isometric force generated by these muscles in common shoulder disorders, i.e. subacromial impingement, calcific tendinitis and grade 2 SS full thickness tears.

In most of the tests we found the expected lower profiles of the torque-time curves of all the tested rotator cuff muscles in comparison to the normal values. Previously the main cause for the decrease in the rotator cuff muscles isometric strength in the presence of intrinsic pathology, such as tear in SS, was attributed mainly to pain [16]. In the present study this conclusion is partially contradicted by the clear findings of different patterns of the not uniformly decreased build up forces of these muscles among the three tested painful disorders of shoulder.

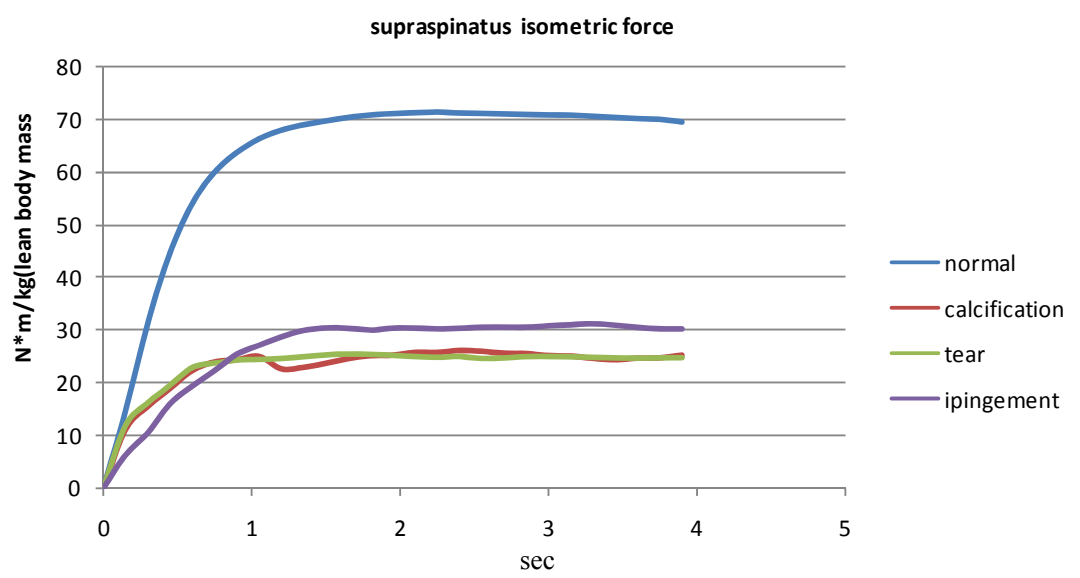

Figure 3. Mean values SS muscle testing normalized to lean body mass from tested individuals with subacromial impingement syndrome (imp) $n=30$, grade 2 tears in SS $n=30$, patients with shoulder calcific tendinitis (calc) $n=30$ and normal individuals (previous data base). The curve profiles differ $(p<0.001)$ in the following order: normal $>>$ imp $>$ tear $=$ calc. 


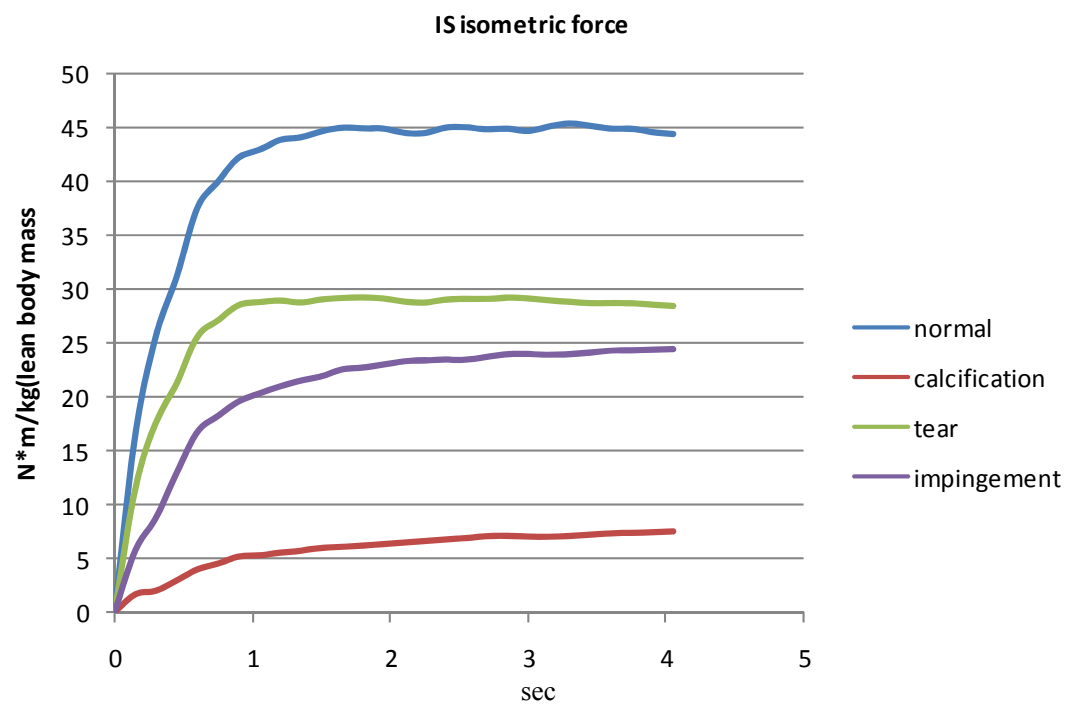

Figure 4. Mean values IS muscle testing normalized to lean body mass from tested individuals with subacromial impingement syndrome (imp) $n=30$, grade 2 tears in SS $n=30$, patients with shoulder calcific tendinitis (calc) $n=30$ and normal individuals (previous data base). The curve profiles differ $(\mathrm{p}<0.001)$ in the following order: normal $>>$ tear $>$ imp $>$ calc.

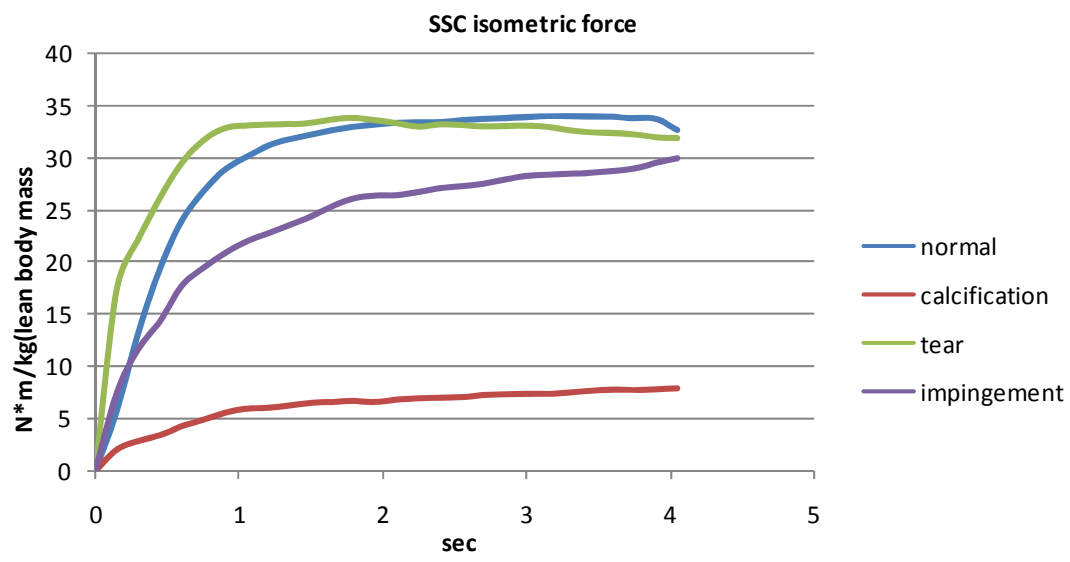

Figure 5. Mean values SSC muscle testing normalized to lean body mass from tested individuals with subacromial impingement syndrome (imp) $n=30$, grade 2 tears in SS $n=30$, patients with shoulder calcific tendinitis (calc) $n=30$ and normal individuals (previous data base). The curve profiles differ $(\mathrm{p}<0.001)$ in the following order: normal $=$ tear $>$ imp $>$ calc.

The best resolution between the curves of the different study groups was found in the testing of IF muscles. The synergistic activity of the rotator cuff muscles and of a deltoid muscle masked the expected differences between the curve profiles of patients with SS tears and with calcific tendonitis, when the SS muscle was tested. In this comparison the curves' profiles of the groups with intrinsic pathology of the SS, e.g. inflammatory in calcific tendinitis and structural in the case of tears, were similar and clearly distinguishable from the group with mostly extrinsic pathology of subacromial impingement. The reason for the similarity of the isometric torque-time curves of SSC muscles in patients with SS tears and in normal population is probably due to the structural distance of the SS and SSC muscles, in a contrast to the structural proximity of SS and IS muscles. Therefore this might be a reason to the sensitivity of the IS muscle testing in the SS intrinsic and extrinsic pathologies, as mentioned above, and relatively low response of SSC muscle to these pathologies of SS. But since the inflammatory process of a calcific tendinitis is not restricted solely to SS and involves also the SSC muscles, causing an intrinsic pathology in SSC, the response of the isometric force testing of SSC in patients with calcific tendinitis indeed reflects this disorder, as expressed by the significantly lower profiles of isometric force generation curve of 
SSC.

In the present study we determined a basis for the future development of muscle testing for diagnosis of disabilities in rotator cuff muscles, i.e. following the recognition of the normal values of the rotator cuff muscles' isometric strength we identified the abnormal patterns of muscle isometric strength in patients with common shoulder rotator cuff disorders. Recognition of these patterns provides a reliable tool for a development of an effective strength testing method for diagnosis of disorders in the rotator cuff. From our results is apparent that the best resolution between the isometric torque-time curves in patients with intrinsic and extrinsic disorders of the rotator cuff muscles is detected in the testing of the IS muscle. Therefore we suggest that mechanical evaluation of the IS muscle, using the presented method, should be utilized for the diagnosis of the shoulder rotator cuff disorders.

\section{REFERENCES}

[1] Jerosch, J., Muller, T. and Castro, W.H. (1991). The incidence of rotator cuff rupture. An anatomic study. Acta Orthopaedica Belgica, 57, 124-129.

[2] Lehman, C., Cuomo, F., Kummer, F.J. and Zuckerman, J.D. (1995) The incidence of full thickness rotator cuff tears in a large cadaveric population. Bulletin of the Hospital for Joint Diseases, 54, 30-31.

[3] Milgrom, C., Schaffler, M., Gilbert, S. and van Holsbeeck, M. (1995) Rotator-cuff changes in asymptomatic adults. The effect of age, hand dominance and gender. The Journal of Bone \& Joint Surgery, 77, 296-298.

[4] Sher, J.S., Uribe, J.W., Posada, A., Murphy, B.J. and Zlatkin, M.B. (1995) Abnormal findings on magnetic resonance images of asymptomatic shoulders. The Journal of Bone \& Joint Surgery of America, 77, 10-15.

[5] Hayes, K., Walton, J.R., Szomor, Z.L. and Murrell, G.A. (2002) Reliability of 3 methods for assessing shoulder strength. Journal of Shoulder and Elbow Surgery, 11, 33-39. doi: $10.1067 / \mathrm{mse} .2002 .119852$

[6] Hughes, R.E. and An, K.N. (1996) Force analysis of rotator cuff muscles. Clinical Orthopaedics and Related Research, 330, 75-83.

doi:10.1097/00003086-199609000-00010

[7] Jenp, Y.N., Malanga, G.A., Growney, E.S. and An, K.N. (1996) Activation of rotator cuff in generating isometric shoulder rotation torque. The American Journal of Sports Medicine, 24, 477-485. doi: $10.1177 / 036354659602400412$

[8] Stoll, T., Huber, E., Seifert, B., Stucki, G. and Michel, B.A. (2002) Isometric muscle strength measurement. Thieme, Stuttgart, New York, 35-37.

[9] Backman, E., Johansson, V., Hager, B., Sjoblom, P. and Henriksson, K.G. (1995) Isometric muscle strength and muscular endurance in normal persons aged between 17 and 70 years. Scandinavian Journal of Rehabilitation Medicine, 27, 109-117.

[10] Hughes, R.E., Johnson, M.E., O’Driscoll, S.W. and An, K.N. (1999) Age related changes in normal isometric shoulder strength. The American Journal of Sports Medicine, 27, 651-657.

[11] Kim, H.M., Teefey, S.A., Zelig, A., Galatz, L.M., Keener, J.D. and Yamaguchi, K. (2009) Shoulder strength in asymptomatic individuals with impact compared with torn rotator cuffs. The Journal of Bone \& Joint Surgery of America, 91, 289-296. doi:10.2106/JBJS.H.00219

[12] Schezar, A, Berkoviz, Y., Haddad, M., Soudry, M. and Rosenberg, N. (2013) Normal isometric strength of rotator cuff muscles in adults. Bone Joint Research, in press.

[13] Neer, C.S. (1983) Impingement lesions. Clinical Orthopaedics and Related Research, 173, 70-77.

[14] Milgrom, C., Schaffler, M., Gilbert, S. and van Holsbeeck, M. (1995) Rotator-cuff changes in asymptomatic adults. The effect of age, hand dominance and gender. The Journal of Bone \& Joint Surgery of Britain, 77, 296-298.

[15] Cofield, R.H. (1982). Subscapular muscle transposition for repair of chronic rotator cuff tears. Surgery Gynecology \& Obstetrics, 154, 667-672.

[16] Steenbrink, F., de Groot, J.H., Veeger, H.E., Meskers, C.G., van de Sande, M.E. and Rozing, P.M. (2006). Pathological muscle activation patterns in patieants with massive rotator cuff tears, with and without subacromialanaesthetics. Manual Therapy, 11, 231-237. doi:10.1016/i.math.2006.07.004

[17] Kuhlman, J.R., Iannoti, J.P., Kelly, M.J., Riegler, F.X, Gevaert M.L. and Ergin, T.M. (1992) Isokinetic and isometric measurement of strength of external rotation and abduction of the shoulder. The Journal of Bone \& Joint Surgery of America, 74, 1320-1333.

[18] Bankes, M.J., Crossman, J.E. and Emery, R.J.H. (1998) A standard method of shoulder strength measurement for the Constant score with a spring balance. Journal of Shoulder and Elbow Surgery, 7, 116-121. doi:10.1016/S1058-2746(98)90220-8

[19] Hallynck, T.H., Soep, H.H., Thomis, J.A., Boelaert, J., Daneels, R. and Dettli, L. (1981). Should clearance be normalized to body surface or to lean body mass? British Journal of Clinical Pharmacology, 11, 523-526. doi:10.1111/j.1365-2125.1981.tb01163.x

[20] Bandholm, T., Rasmussen, L., Aagaard, P., Jensen, B.R. and Diederichsen, L. (2006) Force steadiness, muscle activity, and maximal muscle strength in subjects with subacromial impingement syndrome. Muscle Nerve, 34, 631639. doi: $10.1002 /$ mus. 20636

[21] Kolber, M.J., Beekhuizen, K., Cheng, M.S.S. and Fiebert, I.M. (2007) The reliability of hand-held dynamimetry in measuring isometric strength of the shoulder internal and external rotator musculature using a stabilizing device. Ph Theory Practice, 23, 119-124.

doi:10.1080/09593980701213032 